\title{
International Comparison for RF Power in the Frequency Range up to $18 \mathrm{GHz}$
}

Jan P. M. de Vreede, Willemien Korfage, Patrik Persson, Luciano Brunetti, Valentin Lopez, Ivan Petras, Pierre Morard, Frantisek Hejsek, András Török, Jürgen Rühaak, John Ascroft, Erik Dressler, Murat Celep, Rado Lapuh, Member, IEEE, and Joseph Achkar

\begin{abstract}
A Euromet international laboratory comparison (Project 393) has been carried out between 14 national standards laboratories. Thermistor mounts were used, equipped with PC7 as well as Type $\mathbf{N}$ connectors. The comparison is carried out using the normal equipment of the laboratory for high-quality external calibration. The results show good agreement in measuring the calibration coefficient of the thermistor mounts within the claimed expanded uncertainty (typically between $1 \%$ and $2 \%$ ). It confirms the equivalence of national standards for $\mathrm{RF}$ power up to $18 \mathrm{GHz}$. In one case, corrective action is proposed.
\end{abstract}

Index Terms-Equivalence of standards, measurement standards, power measurement, thermistors, transfer standards, uncertainty.

\section{INTRODUCTION}

A MONG the Euromet HF experts the philosophy was put forward that for transferring the quality of the national standards to the national industry a comparison on the level of routine calibration is very useful in showing coherence of the (inter)national metrology infrastructure. Hence, during their 1997 meeting in Torino, Italy, such a comparison was initiated and registered as Euromet Project 393. It aims to check the quality of measuring the calibration factor $(\mathrm{CF})$ of thermistor mounts in a way similar to that for "high-level customers." This

Manuscript received May 14, 2000; revised October 27, 2000.

J. P. M. de Vreede and W. Korfage are with NMi Van Swinden Laboratorium (VSL), 2600 AR Delft, The Netherlands.

P. Persson is with Celsius Metech AB, S-73226 Arboga, Sweden.

L. Brunetti is with IEN "Galileo Ferraris" (IENGF), I-10135 Torino, Italy.

V. Lopez is with the Instituto National de Tecnica Aerospacial (INTA), 28850 Torrejon de Ardoz, Spain.

I. Petras is with the Slovak Institute of Metrology, Bratislava (SMU), 84255 Slovak Republic.

P. Morard is with the Swiss Federal Office of Metrology (OFMET), CH-3003 Wabern-Bern, Switzerland.

F. Hejsek is with the Czech Metrological Institute (CMI), 10200 Prague, Czech Republic.

A. Török is with the National Office of Measures (OMH), H-1535 Budapest, Hungary.

J. Rühaak is with the Physikalisch-Technische Bundesanstalt (PTB), D-38116 Braunschweig, Germany.

J. Ascroft is with the National Physical Laboratory (NPL), Teddington, U.K.

E. Dressler is with the National Metrology Laboratory (CSIR-NML) TW11 OLW, Pretoria, South Africa.

M. Celep is with the Ulusal Metroloji Enstitüsü (UME), 41470 Gebze, Turkey.

R. Lapuh is with the Slovenian Institute of Quality and Metrology (SIQ), 1000 Ljubljana, Slovenia

J. Achkar is with the Bureau National de Métrologie - Laboratoire Central des Industries Electriques (BNM-LCIE), 92260 Fontenay-aux-Roses, France.

Publisher Item Identifier S 0018-9456(01)02591-8. paper presents the view of the participants after discussing a confidential draft report.

\section{TRAVELING StANDARDS}

To avoid accidental mishaps, it was decided to use two thermistor mounts. In order to check the capability for different connector types, one device is equipped with a PC7-connector and the other one with a Type-N male connector. It is assumed that external customers will usually submit thermistor mounts equipped with Type-N connectors for calibration and only occasionally thermistor mounts with PC7 connector. Therefore, in addition an adapter from PC7 to Type- $\mathrm{N}$ male was added to allow each laboratory to measure at least two devices. Two national standards laboratories each provided a Hewlett Packard Model 8478B thermistor. The pilot laboratory provided the adapter which is part of a normal calibration kit.

In this paper, reference is made to three devices under test (DUT) on which results were obtained, viz. the thermistor mount with Type- $\mathrm{N}$ connector (Code TM1), the thermistor mount with PC7-connector (Code TM2), and the same thermistor mount but now with a PC7-N adapter attached (Code TM3).

\section{Participants And Time Schedule}

NMi Van Swinden Laboratorium (VSL), The Netherlands, acted as coordinator and pilot laboratory for this interlaboratory comparison.

The traveling scheme consisted of four loops (see affiliations of the authors for the full name of the laboratories):

$$
\begin{aligned}
& \text { loop } 1: \text { VSL } \rightarrow \text { Celsius Metech (Sweden) } \rightarrow \text { IENGF (Italy) } \\
& \rightarrow \text { INTA (Spain) } \rightarrow \text { VSL } \\
& \text { loop 2: VSL } \rightarrow \text { SMU (Slovakia) } \rightarrow \text { OFMET (Switzerland) } \\
& \rightarrow \text { CMI (Czech Republic) } \rightarrow \text { OMH (Hungary) } \rightarrow \text { VSL } \\
& \text { loop 3: VSL } \rightarrow \text { PTB (Germany) } \rightarrow \text { NPL (U.K.) } \rightarrow \text { VSL } \\
& \text { loop 4: VSL } \rightarrow \text { CSIR (South Africa) } \rightarrow \text { UME (Turkey) } \rightarrow \\
& \text { SIQ (Slovenia) } \rightarrow \text { BNM-LCIE (France) } \rightarrow \text { VSL },
\end{aligned}
$$

starting in February 1998 and finishing in July 1999 at the pilot laboratory. In the original circulation scheme, three weeks per laboratory was allowed for measurement and transport. The measurements were carried out in a period only about two months longer than planned, using some minor modifications in the schedule. Reporting, however, took much longer than was foreseen originally: from 20 to 220 days, with a median of 50 days (the protocol requested a maximum of 30 calendar days). 


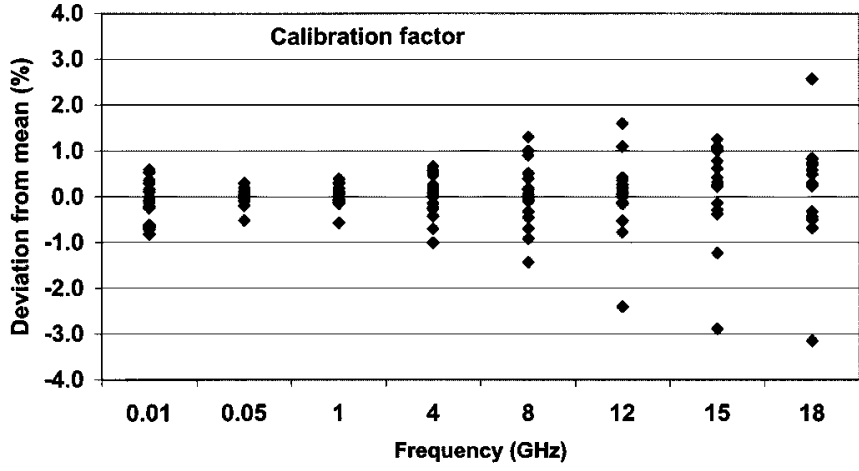

Fig. 1. Results from all participants on the measurement of the $\mathrm{CF}$ of thermistor mount TM1 for all eight frequencies. The results are presented as deviations from the reference value; no uncertainty bars are indicated.

\section{PROTOCOL FOR THE COMPARISON}

In this comparison, an attempt is made to implement the guidelines for key comparisons [1]. Hence, the pilot laboratory composed a protocol, in which participants were asked to present their results on the $\mathrm{CF}$ and the magnitude of the reflection coefficient at eight frequencies, including a statement of uncertainty with a coverage factor of $k=2$. Furthermore, a detailed uncertainty budget was requested for information about the important contributions. Guidance for providing such an uncertainty budget was given by referring to an example given in [2]. In addition the traceability for the standards used was requested to check on correlation between measurement results.

A number of the laboratories did not submit a detailed uncertainty budget at the time of reporting and had to be reminded to provide this information. After receipt of additional information the pilot laboratory prepared a first version of the so-called Draft A report for discussion during the Euromet HF Meeting in March 2000.

\section{MEASUREMENT SET-UPS}

The protocol stipulated that the laboratory should apply the same measurement instrumentation as in use for high-level calibration for external customers. Therefore, only PTB used its primary facility: a microcalorimeter system. All other systems are based on a (in)direct comparison between a (working) standard and the DUT.

In this section, some characteristic details of the measurement set-up are given, including the traceability

Celsius: A power splitter system is used with a feedback via the monitoring arm to keep the power into the second arm constant. The standard (a thermo-electric sensor) is calibrated at SESC (U.K.) a UKAS accredited calibration laboratory.

IENGF: A power splitter system is used with a $6 \mathrm{~dB}$ attenuator attached to the test port to improve the VSWR of the system. The ratio between the responses of the power readings on both arms is obtained as measurement value. The standard is a thermistor mount and traceable to the IEN primary facility (a microcalorimeter).
INTA: A feedthrough system is used which is calibrated on a regular basis using thermistor mounts traceable to NIST. The system provides a constant output power due to a feedback loop using a feedthrough mount of which the housing is temperature controlled.

$S M U$ : A feedthrough system is used which provides a calibrated output power (feedback via a directional coupler). The standard is traceable to the SMU microcalorimeter. OFMET: A power splitter system is used with the standard (a thermistor mount) on one arm and the DUT on the other. The standard is traceable to NMi VSL.

CMI: A power splitter system is used with a feedback via the monitoring arm to keep the power into the second arm constant. Standard and DUT are placed alternately on this arm, and are of similar design (thermistor mounts). The standard is traceable to PTB.

$O M H$ : A feedthrough system is used for which the output level is monitored via a directional coupler. An attenuator is used to improve the VSWR of the system. The standard and DUT are placed alternatively on the test port. The standard is a thermistor mount and traceable to the $\mathrm{OMH}$ primary facility (a microcalorimeter); for $10 \mathrm{MHz}$ a calorimetric device is used. The same bridge (HP 432B with external DVM) is used for standard and DUT.

PTB: The measurements are performed in the PTB primary facility (a microcalorimeter).

$N P L$ : Two different systems are used:

- Up to $8 \mathrm{GHz}$ : a power splitter system is used with a monitoring sensor in one arm. The ratio between the responses of the power readings on both arms is obtained as the measurement value. The standard is a coaxial calorimeter and is traceable to the NPL primary facility (a $14 \mathrm{~mm}$ dry twin calorimeter).

- Above $8 \mathrm{GHz}$ : a multistate reflectometer is used which includes a monitor for power output. The measurand is the ratio of the power readings (after normalization using the monitor signal) between the standard (including a waveguide-to-coax adapter) and the DUT which are attached alternately to the test port. The waveguide standards are traceable to the NPL primary facilities (microcalorimeters).

CSIR-NML: A power splitter system is used which is calibrated on a regular basis using the NML primary facility (a dry twin-load calorimeter) and a monitoring sensor. The ratio between the responses of the power readings on both arms is obtained as measurement value. In this way, a relation is obtained between the power reading of a monitoring sensor in one arm and the output power in the other arm.

$U M E$ : The measurement system consists of a stable signal generator system (containing an amplifier and an adapter in addition). The standard and DUT are placed alternately on the output port of the generator, and are of similar design (thermistor mounts). Traceability is based on the primary UME facility (microcalorimeter) and a comparison with LCIE on that level.

SIQ: A power splitter system is used which is calibrated on a regular basis using power sensors which are traceable to SESC (U.K.). The standard is used as monitoring sensor. 


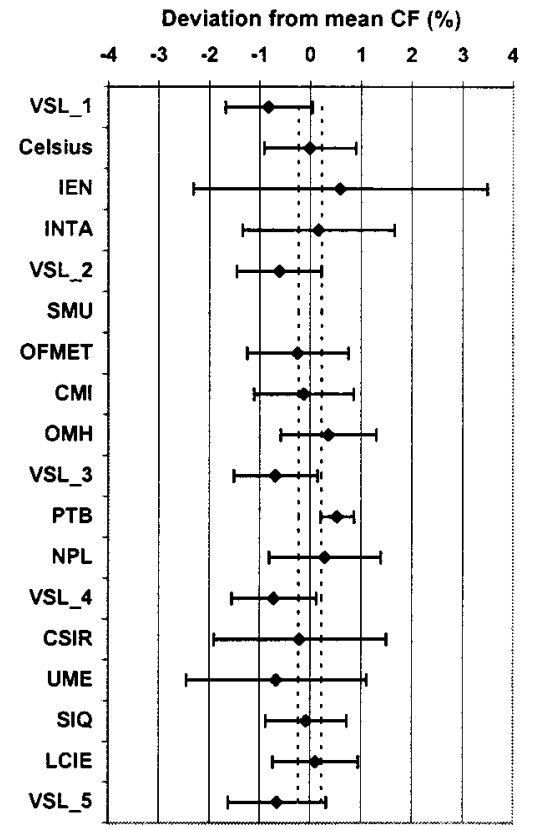

(a)

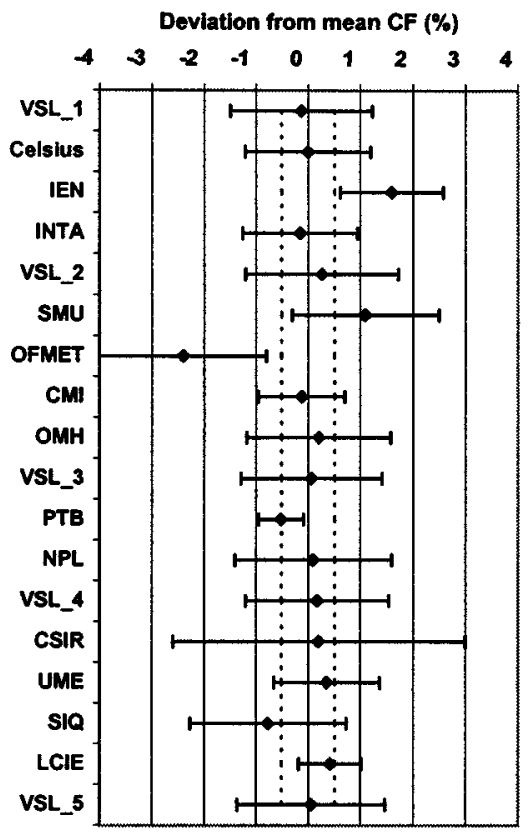

(c)

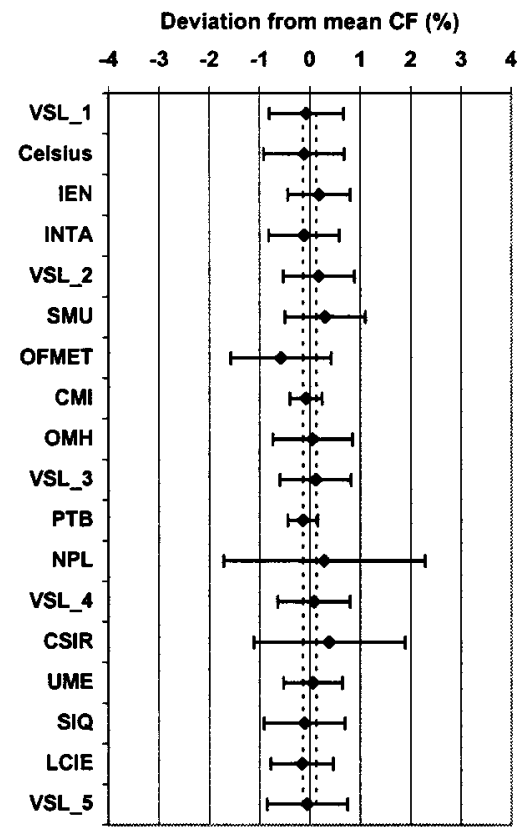

(b)

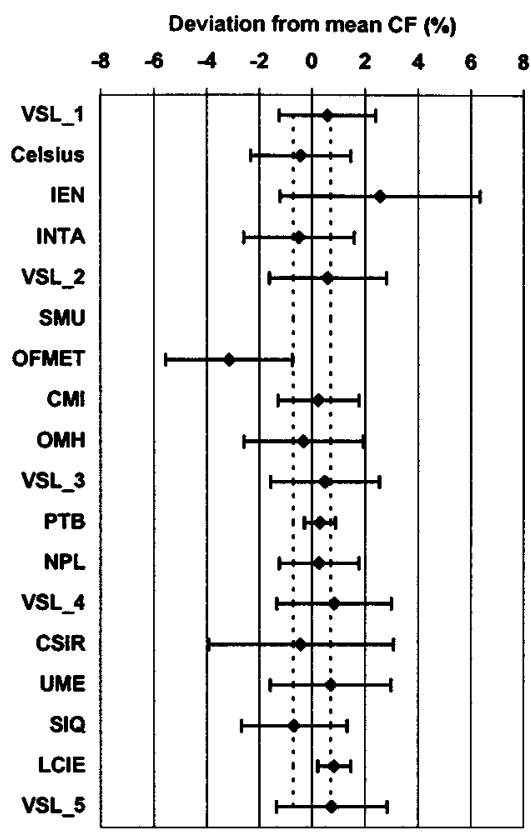

(d)

Fig. 2. Results on the measurement of the CF of thermistor TM1 for four frequencies (a): $10 \mathrm{MHz}$; (b): $1 \mathrm{GHz}$; (c): $12 \mathrm{GHz}$; and (d): $18 \mathrm{GHz}$, as obtained by each participant. The uncertainty bars refer to an uncertainty with a coverage factor of $k=2$.

BNM-LCIE: Two systems are used:

- Below 1 GHz: a calibrated power splitter system is used.

- $1 \mathrm{GHz}$ and higher: a calibrated six-port system is used. Both systems have an output port equipped with PC7-connector. A suitable adapter (PC7 to N-female) is used to measure TM1. The standards (with PC7connector) are traceable to the LCIE primary facility (microcalorimeter).

NMi VSL: The measurement system consists of a stable signal generator, with a $10 \mathrm{~dB}$ attenuator to improve the VSWR of the output port. The standard and DUT are placed alternately on the output port of the generator, and are of similar design (thermistor mounts). Traceability is based on the primary VSL facility (microcalorimeter).

\section{MeAsurement Results}

The participants were asked to submit measurement results on each thermistor mount at eight frequencies $(10 \mathrm{MHz}$, $50 \mathrm{MHz}, 1 \mathrm{GHz}, 4 \mathrm{GHz}, 8 \mathrm{GHz}, 12 \mathrm{GHz}, 15 \mathrm{GHz}$, and $18 \mathrm{GHz}$ ): both its $\mathrm{CF}$ and its reflection coefficient, with an expanded uncertainty $(k=2)$. 
TABLE I

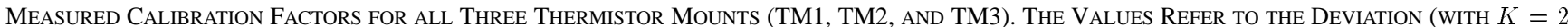

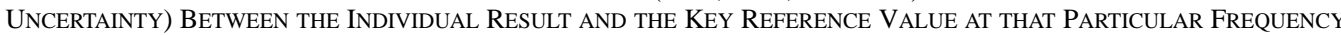

\begin{tabular}{|c|c|c|c|c|c|c|c|c|}
\hline \multicolumn{2}{|c|}{ Thermistor TM1 } & \multicolumn{4}{|c|}{ Frequency in $\mathrm{GHz}$} & \multirow[b]{2}{*}{12.0} & \multirow[b]{2}{*}{15.0} & \multirow[b]{2}{*}{18.0} \\
\hline Laboratory & 0.01 & 0.05 & 1.0 & 4.0 & 8.0 & & & \\
\hline Celsius & $-0.0 \pm 0.9$ & $0.2 \pm 0.7$ & $-0.1 \pm 0.8$ & $0.1 \pm 1.0$ & $-0.1 \pm 1.3$ & $-0.0 \pm 1.2$ & $0.6 \pm 1.3$ & $-0.4 \pm 1.9$ \\
\hline IEN & $0.6 \pm 2.9$ & $-0.1 \pm 0.8$ & $0.2 \pm 0.6$ & $0.3 \pm 0.7$ & $0.9 \pm 1.0$ & $1.6 \pm 1.0$ & $1.0 \pm 0.5$ & $2.6 \pm 3.8$ \\
\hline INTA & $0.2 \pm 1.5$ & $0.0 \pm 0.8$ & $-0.1 \pm 0.7$ & $0.0 \pm 0.9$ & $-0.1 \pm 1.1$ & $-0.2 \pm 1.1$ & $0.3 \pm 1.2$ & $-0.5 \pm 2.1$ \\
\hline SMU & -- & $-0.5 \pm 1.1$ & $0.3 \pm 0.8$ & $-0.7 \pm 0.9$ & $-0.7 \pm 1.2$ & $1.1 \pm 1.4$ & --- & $+\cdots$ \\
\hline OFMET & $-0.2 \pm 1.0$ & $-0.2 \pm 0.9$ & $-0.6 \pm 1.0$ & $-1.0 \pm 1.2$ & $-1.4 \pm 1.5$ & $-2.4 \pm 1.6$ & $-2.9 \pm 1.8$ & $-3.2 \pm 2.4$ \\
\hline CMI & $-0.1 \pm 1.0$ & $-0.0 \pm 0.5$ & $-0.1 \pm 0.3$ & $-0.2 \pm 0.4$ & $-0.5 \pm 0.6$ & $-0.1 \pm 0.8$ & $-0.3 \pm 0.5$ & $0.2 \pm 1.5$ \\
\hline $\mathrm{OMH}$ & $0.4 \pm 0.9$ & $0.1 \pm 0.8$ & $0.1 \pm 0.8$ & $0.5 \pm 1.1$ & $0.0 \pm 1.4$ & $0.2 \pm 1.4$ & $0.2 \pm 0.2$ & $-0.3 \pm 2.3$ \\
\hline PTB & $0.5 \pm 0.3$ & $0.0 \pm 0.3$ & $-0.1 \pm 0.3$ & $-0.4 \pm 0.3$ & $-0.9 \pm 0.4$ & $-0.5 \pm 0.4$ & $-1.2 \pm 0.5$ & $0.3 \pm 0.6$ \\
\hline$N P L$ & $0.3 \pm 1.1$ & $0.3 \pm 1.0$ & $0.3 \pm 2.0$ & $0.5 \pm 2.0$ & $1.3 \pm 2.1$ & $0.1 \pm 1.5$ & $0.4 \pm 1.9$ & $0.3 \pm 1.5$ \\
\hline CSIR & $-0.2 \pm 1.7$ & $0.3 \pm 1.5$ & $0.4 \pm 1.5$ & $0.7 \pm 2.0$ & $1.0 \pm 2.2$ & $0.2 \pm 2.8$ & $-0.4 \pm 3.0$ & $-0.4 \pm 3.5$ \\
\hline UME & $-0.7 \pm 1.8$ & $0.1 \pm 0.5$ & $0.1 \pm 0.6$ & $0.2 \pm 0.8$ & $0.2 \pm 0.9$ & $0.4 \pm 1.0$ & $-0.1 \pm 1.9$ & $0.7 \pm 2.3$ \\
\hline$S I Q$ & $-0.1 \pm 0.8$ & $0.0 \pm 0.8$ & $-0.1 \pm 0.8$ & $0.6 \pm 1.0$ & $0.5 \pm 1.6$ & $-0.8 \pm 1.5$ & $0.2 \pm 2.0$ & $-0.7 \pm 2.0$ \\
\hline LCIE & $0.1 \pm 0.8$ & $-0.2 \pm 0.4$ & $-0.2 \pm 0.6$ & $-0.3 \pm 0.6$ & $-0.3 \pm 0.6$ & $0.4 \pm 0.6$ & $1.1 \pm 0.6$ & $0.8 \pm 0.6$ \\
\hline VSL & $-0.7 \pm 0.9$ & $0.0 \pm 0.6$ & $0.0 \pm 0.7$ & $-0.0 \pm 0.9$ & $0.2 \pm 1.2$ & $0.1 \pm 1.4$ & $1.0 \pm 1.3$ & $0.6 \pm 2.1$ \\
\hline
\end{tabular}

\begin{tabular}{|c|c|c|c|c|c|c|c|c|}
\hline \multicolumn{2}{|c|}{ Thermistor TM2 } & \multicolumn{3}{|c|}{ Frequency in $\mathrm{GHz}$} & \multirow[b]{2}{*}{8.0} & \multirow[b]{2}{*}{12.0} & \multirow[b]{2}{*}{15.0} & \multirow[b]{2}{*}{18.0} \\
\hline Laboratory & 0.01 & 0.05 & 1.0 & 4.0 & & & & \\
\hline Celsius & --- & -- & - & $\ldots$ & --- & -.-- & --- & --- \\
\hline IEN & --- & -- & -- & $\cdots$ & --- & --- & $\ldots$ & --- \\
\hline INTA & $-0.6 \pm 2.5$ & $-0.1 \pm 1.3$ & $-0.1 \pm 1.4$ & $-0.3 \pm 1.5$ & $-0.2 \pm 1.7$ & $0.1 \pm 1.9$ & $0.0 \pm 2.1$ & $0.4 \pm 2.7$ \\
\hline SMU & ... & $\cdots$ & -.. & $\cdots$ & --- & --- & --- & --- \\
\hline OFMET & $-0.1 \pm 0.7$ & $-0.0 \pm 0.6$ & $-0.2 \pm 0.7$ & $-0.3 \pm 0.8$ & $-0.3 \pm 1.2$ & $-1.5 \pm 1.2$ & $-0.6 \pm 1.5$ & $-2.8 \pm 2.1$ \\
\hline $\mathrm{CMI}$ & $\ldots$ & -- & --- & --- & --- & --- & --- & --- \\
\hline $\mathrm{OMH}$ & $0.8 \pm 1.0$ & $0.0 \pm 0.8$ & $-0.1 \pm 0.8$ & $-0.3 \pm 1.1$ & $-0.4 \pm 1.4$ & $0.2 \pm 1.5$ & --- & --- \\
\hline PTB & $0.7 \pm 0.3$ & $-0.0 \pm 0.3$ & $-0.1 \pm 0.3$ & $-0.2 \pm 0.3$ & $-0.4 \pm 0.3$ & $-0.2 \pm 0.4$ & $-0.5 \pm 0.4$ & $-0.7 \pm 0.5$ \\
\hline NPL & $0.4 \pm 2.2$ & $0.2 \pm 2.2$ & $-0.0 \pm 2.8$ & $0.3 \pm 2.8$ & $0.3 \pm 2.9$ & $-0.4 \pm 2.5$ & $0.3 \pm 2.7$ & $-0.0 \pm 2.5$ \\
\hline CSIR & $-0.1 \pm 1.6$ & $0.2 \pm 1.5$ & $0.2 \pm 1.5$ & $0.5 \pm 2.1$ & $0.5 \pm 2.3$ & $0.5 \pm 3.3$ & $-0.4 \pm 3.5$ & $1.7 \pm 4.6$ \\
\hline UME & $-0.6 \pm 1.8$ & $-0.0 \pm 0.5$ & $0.1 \pm 0.7$ & $0.3 \pm 1.3$ & $0.5 \pm 1.7$ & $1.0 \pm 2.8$ & $0.2 \pm 3.5$ & $0.8 \pm 4.2$ \\
\hline SIQ & $\ldots$ & $0.0 \pm 0.8$ & $0.1 \pm 0.8$ & $0.3 \pm 1.0$ & $0.2 \pm 1.4$ & $0.1 \pm 1.5$ & $0.7 \pm 2.0$ & $0.5 \pm 2.1$ \\
\hline LCIE & $0.1 \pm 0.8$ & $-0.3 \pm 0.4$ & $0.1 \pm 0.4$ & $0.2 \pm 0.4$ & $0.3 \pm 0.4$ & $0.2 \pm 0.4$ & $0.4 \pm 0.4$ & $0.8 \pm 0.4$ \\
\hline VSL & $-0.6 \pm 0.7$ & $0.0 \pm 0.4$ & $-0.1 \pm 0.4$ & $-0.4 \pm 0.5$ & $-0.5 \pm 0.7$ & $0.0 \pm 0.8$ & $-0.1 \pm 0.9$ & $-0.7 \pm 1.1$ \\
\hline \multicolumn{2}{|c|}{ Thermistor TM3 } & \multicolumn{3}{|c|}{ Frequency in $\mathrm{GHz}$} & & & & \\
\hline Laboratory & 0.01 & 0.05 & 1.0 & 4.0 & 8.0 & 12.0 & 15.0 & 18.0 \\
\hline Celsius & $-0.1 \pm 0.9$ & $0.1 \pm 0.7$ & $-0.1 \pm 0.8$ & $-0.0 \pm 1.0$ & $-0.3 \pm 1.3$ & $-0.2 \pm 1.2$ & $0.7 \pm 1.4$ & $-0.2 \pm 1.5$ \\
\hline IEN & $0.7 \pm 2.8$ & $-0.3 \pm 0.8$ & $0.0 \pm 0.6$ & $-0.0 \pm 0.5$ & $1.1 \pm 0.6$ & $1.6 \pm 1.3$ & $0.3 \pm 0.7$ & $2.3 \pm 4.1$ \\
\hline INTA & $0.3 \pm 1.5$ & $0.0 \pm 0.8$ & $-0.1 \pm 0.8$ & $0.1 \pm 0.9$ & $-0.1 \pm 1.1$ & $-0.6 \pm 1.1$ & $0.0 \pm 1.3$ & $-0.6 \pm 2.0$ \\
\hline SMU & ---- & $0.1 \pm 1.4$ & $0.2 \pm 1.0$ & $-0.6 \pm 1.1$ & $-0.1 \pm 1.3$ & $1.8 \pm 1.6$ & --- & --- \\
\hline OFMET & $\cdots$ & -- & -- & -.- & --- & --- & --- & -- \\
\hline $\mathrm{CMI}$ & $-0.4 \pm 0.9$ & $-0.2 \pm 0.5$ & $-0.2 \pm 0.3$ & $-0.4 \pm 0.5$ & $-0.7 \pm 0.5$ & $-0.6 \pm 1.0$ & $-0.3 \pm 0.6$ & $-1.7 \pm 2.2$ \\
\hline $\mathrm{OMH}$ & $0.5 \pm 1.0$ & $-0.0 \pm 0.8$ & $-0.2 \pm 0.8$ & $0.3 \pm 1.1$ & $-0.2 \pm 1.4$ & $-0.3 \pm 1.4$ & $-0.5 \pm 2.1$ & $1.0 \pm 2.7$ \\
\hline РТВ & --- & --- & --- & --- & --- & --- & --- & --- \\
\hline NPL & $0.3 \pm 1.1$ & $0.1 \pm 1.0$ & $0.0 \pm 2.0$ & $0.2 \pm 2.0$ & $0.4 \pm 2.0$ & $-0.4 \pm 2.0$ & $0.3 \pm 2.0$ & $-0.9 \pm 2.0$ \\
\hline CSIR & $-0.2 \pm 1.6$ & $0.1 \pm 1.5$ & $0.2 \pm 1.5$ & $0.7 \pm 1.9$ & $0.5 \pm 2.0$ & $0.4 \pm 2.8$ & $-0.6 \pm 2.9$ & $0.9 \pm 4.0$ \\
\hline UME & $-0.6 \pm 1.8$ & $0.1 \pm 0.5$ & $0.1 \pm 0.5$ & $0.0 \pm 0.8$ & $0.0 \pm 0.6$ & $0.0 \pm 1.6$ & $-0.4 \pm 3.0$ & $-0.4 \pm 3.2$ \\
\hline SIQ & $-0.2 \pm 0.8$ & $-0.0 \pm 0.8$ & $-0.1 \pm 0.8$ & $-0.1 \pm 1.0$ & $-0.4 \pm 1.4$ & $-1.2 \pm 1.5$ & $-0.5 \pm 2.0$ & $-0.2 \pm 2.0$ \\
\hline LCIE & -.- & --- & --- & --- & $\ldots$ & -.-- & $\ldots$ & $\ldots$ \\
\hline VSL & $-0.6 \pm 0.9$ & $-0.0 \pm 0.6$ & $0.1 \pm 0.8$ & $-0.1 \pm 0.9$ & $0.0 \pm 1.2$ & $-0.3 \pm 1.5$ & $0.8 \pm 1.5$ & $0.1 \pm 2.0$ \\
\hline
\end{tabular}

The mathematical average of the measurement values on the $\mathrm{CF}$ and the reflection factor is calculated for each of the three devices using an equal weight for each laboratory. The partic- ipants decided that these average values should be used as the reference value for each DUT. As the actual value is specific for each device itself and not for the laboratory's facility, it is 
better to look to the deviations from the mean value, and to make the key reference effectively equal to zero. The results from all DUTs can then be compared against a common reference line. The uncertainty in the reference value is calculated as the uncertainty $(k=2)$ in the mean of the measurement data assuming a normal distribution.

The results obtained at VSL over a period of 17 months indicate that no significant change in the DUTs has occurred within the uncertainty.

The actual results on the CF for all DUTs and for all frequencies are given in Table I. The normal practice of expressing CF in a percentage value is used. The deviations given are obtained by subtracting the calculated mean from the measured CF. The uncertainties as given in Table I are calculated as the RSS of the measurement uncertainty and the uncertainty in the calculated mean.

In the figures, examples are given for one DUT only, but they are typical for all DUTs. In Fig. 1, an overview is given to indicate the spread in the measured values for the CF of TM1 for all eight frequencies. In Fig. 2, the results on the CF of TM1 are presented for four frequencies and for all laboratories explicitly, together with uncertainty bars for $k=2$.

The reflection coefficient is considered to be an auxiliary parameter, mainly needed to determine the mismatch uncertainty. In Fig. 3, an overview is given for TM1 as a typical example.

\section{ANALYSIS OF THE RESUltS}

As usual in international comparisons the amount of information is quite dependent upon the specific laboratory. Aside from contribution in the budget due to the specific measurement set-up most laboratories indicate the following four main contributions:

- Uncertainty in reference standard

- Mismatch signal source-reference standard

- Mismatch signal source-DUT

- Reproducibility (spread in the measurement data)

Usually the laboratory reference standard contributed more than $50 \%$ to the uncertainty in the value of the CF.

Two laboratories provided a much smaller uncertainty than the other participants for the measured CF: PTB due to the use of a primary facility, and BNM-LCIE due to a small uncertainty for its reference standard.

As the mean value strongly depends on the results from the other participants, laboratories claiming a small uncertainty might get inconsistent results, i.e., the difference between their result and the reference value is larger than the combined value of their claimed uncertainty and the uncertainty in the key reference value. The uncertainty of the latter is in most cases of the order of $0.4 \%$, but not larger than $0.8 \%$. Such an inconsistency has occurred accidentally, see PTB and BNM-LCIE results above $12 \mathrm{GHz}$.

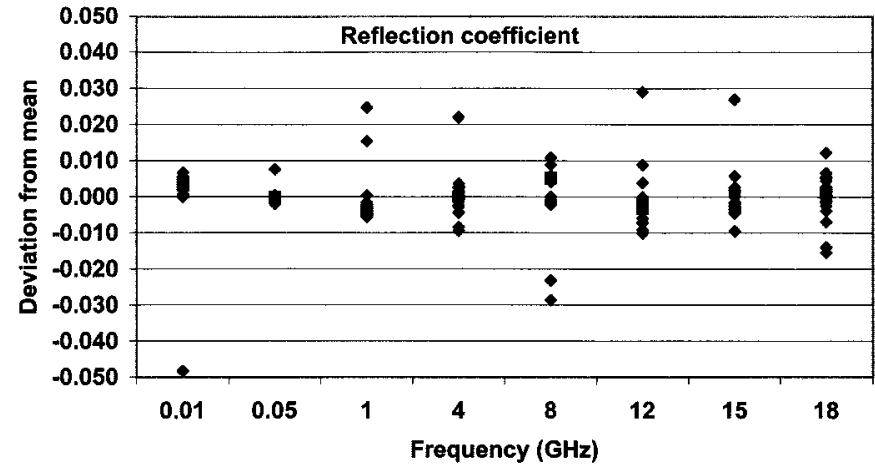

Fig. 3. Results from all participants on the measurement of the reflection coefficient of thermistor mount TM1 for all eight frequencies. The results are presented as deviations from the reference value; no uncertainty bars are indicated.

In addition some inconsistencies are present when comparing the results between pairs of laboratories. No action is foreseen unless further analysis reveals a clear source for these inconsistencies.

Only the results from OFMET show a clear trend deviating from the others at higher frequencies. Those measurements were carried out before the group moved into the OFMET buildings. As they are now also using different equipment, it is impossible to reproduce the measurement conditions. A follow-up comparison is proposed to clarify this discrepancy.

For the reflection coefficient discrepancies are observed as well. In the present framework the influences on the $\mathrm{CF}$ due to such discrepancies is rather small (of the order of $0.1 \%$ ). However, in a comparison dedicated to impedance further investigation into the uncertainty budget is recommended.

\section{CONCLUSIONS}

The stated uncertainty for the $\mathrm{CF}$ ranges from $0.3 \%$ at $50 \mathrm{MHz}$ to more than $4.0 \%$ at $18 \mathrm{GHz}$, independent of the type of connector on the DUT. Almost all results are consistent within the claimed uncertainty, except for one laboratory, for which a new small-scale comparison should be organized to investigate the problem. The uncertainty stated for the reflection coefficient was up to 0.03 in almost all cases. Most of the results are consistent within the claimed uncertainty.

The results support the equivalence of national standards laboratories for the measurement of the $\mathrm{CF}$ of thermistor mounts based upon methods routinely used in calibrations for external customers.

\section{REFERENCES}

[1] (2000, Mar.) Guidelines on Key Comparisons, Version 1. [Online]. Available: http://www.bipm.fr, directory:enus/8_key_comparisons/guidelines.html

[2] (1999, Dec.) Expressions of the Uncertainty of Measurement in Calibration, Document EA-04/02. [Online]. Available: http://www.europeanaccreditation.org, Directory: Documents 